\title{
Robust Model Predictive Control for an Uncertain Smart Thermal Grid
}

\author{
Samira S. Farahani*, Zofia Lukszo**, Tamás Keviczky***, Bart De Schutter***, Richard M. Murray*
}

\begin{abstract}
The focus of this paper is on modeling and control of Smart Thermal Grids (STGs) in which the uncertainties in the demand and/or supply are included. We solve the corresponding robust model predictive control (MPC) optimization problem using mixed-integer-linear programming techniques to provide a day-ahead prediction for the heat production in the grid. In an example, we compare the robust MPC approach with the robust optimal control approach, in which the day-ahead production plan is obtained by optimizing the objective function for entire day at once. There, we show that the robust MPC approach successfully keeps the supply-demand balance in the STG while satisfying the constraints of the production units in the presence of uncertainties in the heat demand. Moreover, we see that despite the longer computation time, the performance of the robust MPC controller is considerably better than the one of the robust optimal controller.
\end{abstract}

\section{INTRODUCTION}

Smart Thermal Grids (STGs) can contribute to obtaining sustainable energy systems by guaranteeing a reliable heating supply to various customers by using renewable energy sources such as solar or geothermal energy. STGs are best applicable to neighborhoods with small-scale utility companies and independent users. As about half of a neighborhood's electricity consumption is typically used for thermal purposes [1], introducing STG neighborhoods could have substantial benefits, such as: 1) less transport of energy, less energy loss, and lower transportation costs, and 2) using the produced heat at the neighborhood level as an energy source to avoid wasting heat.

Considering the complexities of such systems, mainly due to uncertainty in demand and supply as well as the large size of the networks, smart energy systems need to be managed and controlled in an automated way in order to increase the efficiency for both producers and consumers. To this end, model predictive control (MPC) [15] has been proved to be a useful tool in both simulations and real-life applications [7], [12], [13].

We consider robust MPC for STGs in the presence of uncertainties in the grid to provide a day-ahead heat production plan for the thermal grid. The uncertainties in the network can be due to the uncertainty in the demand and/or in the production because of using different resources such as solar energy or biogas. Although the control aspects of thermal energy have been studied implicitly in the context of

${ }^{*}$ Samira S. Farahani and Richard M. Murray are with the Department of Control and Dynamical Systems, California Institute of Technology, Pasadena, CA. farahani@caltech.edu

**Zofia Lukszo is with the Energy and Industry Division, Delft University of Technology, Delft, the Netherlands

*** Tamás Keviczky and Bart De Schutter are with the Delft Center for Systems and Control, Delft University of Technology, Delft, the Netherlands
Combined Heat and Power (CHP) systems or general smart grids, using distributed MPC and other similar agent-based control approaches [9], [14], the explicit implementation of the controller for STG systems requires careful investigation due to the structural differences between STGs and other types of smart grids such as smart electric grids. In the context of smart thermal grids and MPC, to the authors' best knowledge, this paper will be the first attempt that studies robust MPC for STGs. Hence, the novelty of our contribution is not in the approach but is in the application of this approach. To model the network, we use a mixed logical dynamical (MLD) model and we assume that the uncertainty is bounded within a polyhedral set. Hence, the worst-case MPC optimization problem can be recast as a Mixed-Integer-Linear Programming (MILP) problem which can be solved efficiently using the available algorithms. In the end, in an example, we compare this approach with the robust optimal control approach in which the optimal input is calculated at once for the entire simulation hours (24 hours related to the day-ahead prediction).

\section{SMART THERMAL GRIDS}

We consider a regional network of greenhouses, which is a typical example of a thermal grid. Each of these greenhouses is considered as an agent and the full information of each agent, such as the production resources, the demand request for the next day, etc., is assumed to be available to the whole network. Each agent is facilitated with an CHP system and a boiler and hence, is capable of local production of heat and electricity that can be used by the same agent or be exported/sold to the network. We assume that the agents can only trade heat among each other and the electricity will be bought or sold to the electricity market only. Moreover, each agent has a buffer system to store heat and either to use it internally or to sell it to the other agents in the network. In addition to the local heat generation, there are one or more external parties that can provide heat to the network. We consider all the external parties as one single agent. The greenhouses are connected to each other and to the external suppliers by several pipes of different sizes. Moreover, to adjust the input and output heat to and from the greenhouses, there are several heat exchangers located outside the greenhouses.

To model the physical system, we discretize the system with sampling time of one hour. The time step counter is denoted by $k$. For the sake of compactness, the definition of model parameters are presented in Table I.

As indicated by experts, the heat exchangers do not add additional costs to the heat production and hence, they can 


\begin{tabular}{|l|l|l|}
\hline Parameters & Symbol & Unit \\
\hline Transportation cost per MW & $C_{\text {trans }}$ & $€$ \\
The energy content of gas for CHP start up & $g_{\text {start }}$ & $\mathrm{MW}$ \\
Electrical efficiency of the CHP unit & $\eta_{\mathrm{e}}$ & - \\
Thermal efficiency of the CHP unit & $\eta_{\text {th }}$ & - \\
Thermal efficiency of the boiler & $\eta_{\text {Boil }}$ & - \\
Turnaround efficiency of the buffer unit & $\eta_{\text {Buf }}$ & - \\
Fuel price per MW & $F_{\text {price }}$ & $€$ \\
CHP maintenance cost per MW & $C_{\mathrm{CHP}}$ & $€$ \\
CHP fixed start up cost & $C_{\text {fix }}$ & $€$ \\
Buffer capacity of each greenhouse & $B_{\mathrm{C}}$ & $\mathrm{MWh}$ \\
Minimum heat production capacity for unit $u$ & $\mathrm{U}_{u}$ & $\mathrm{MWh}$ \\
Maximum heat production capacity for unit $u$ & $\mathrm{U}_{u}$ & $\mathrm{MWh}$ \\
\hline
\end{tabular}

TABLE I

be left out from the network model. The fuel energy content (gas in our case) used by a CHP unit at greenhouse $j$ at time step $k$ in MW can be specified as [16]

$$
g_{\mathrm{CHP} j}(k)=\frac{P_{\mathrm{G}_{\mathrm{CHP}} j}(k)}{\eta_{\mathrm{e}}}=H_{\mathrm{G}_{\mathrm{CHP}}}(k) \cdot \frac{1}{\eta_{\mathrm{th}}},
$$

where $P_{\mathrm{G}_{\mathrm{CHP}} j}(k)$ and $H_{\mathrm{G}_{\mathrm{CHP}} j}(k)$ are respectively the electrical power and the heat generated by the CHP unit of greenhouse $j$ at time step $k$ in MW. Similarly, for a boiler, we have [10]

$$
g_{\mathrm{Boil} j}(k)=H_{\mathrm{G}_{\mathrm{Boil}} j}(k) \cdot \frac{1}{\eta_{\mathrm{Boil}}},
$$

where $g_{\mathrm{Boil} j}(k)$ is defined similarly to $g_{\mathrm{CHP} j}(k)$ and $H_{\mathrm{G}_{\mathrm{Boil}} j}(k)$ is the heat generated by the boiler of greenhouse $j$ at time step $k$ in MW.

If the CHP or boiler unit are operating at greenhouse $j$, the thermal power can vary at each time step between a certain minimum and maximum for both the CHP and the boiler as

$$
\begin{aligned}
& \underline{\mathrm{U}}_{\mathrm{CHP} j} \leq H_{\mathrm{G}_{\mathrm{CHP}} j}(k) \leq \overline{\mathrm{U}}_{\mathrm{CHP} j} \quad \forall k, j \\
& \underline{\mathrm{U}}_{\mathrm{Boil} j} \leq H_{\mathrm{G}_{\mathrm{Boil}} j}(k) \leq \overline{\mathrm{U}}_{\mathrm{Boil} j} \quad \forall k, j .
\end{aligned}
$$

Moreover, in the case that the production units, i.e., the boiler and the CHP, of greenhouse $j$ produce more heat than is demanded by the greenhouse itself, the heat can be stored in a buffer to be used at other hours or to be used by other greenhouses in the network. We assume that each greenhouse $j$ can only send or receive heat to or from its immediate neighbors, respectively. Let $H_{\text {exchij }}$ denotes the exchanged heat between two adjacent greenhouses $i$ and $j$. The buffer state of greenhouse $j$ can then be defined as

$$
\begin{aligned}
B_{\mathrm{S} j}(k) & =B_{\mathrm{S} j}(k-1)+\eta_{\mathrm{Buf}}\left(H_{\mathrm{G}_{\mathrm{CHP}} j}(k)+H_{\mathrm{G}_{\mathrm{Boil}} j}(k)\right. \\
& \left.-H_{\mathrm{D} j}(k)+H_{\mathrm{impEx} j}(k)+\sum_{i \in \phi_{j}}\left(1-\alpha_{i j}\right) H_{\mathrm{exchij}}\right),
\end{aligned}
$$

where $H_{\mathrm{D} j}(k)$ denotes the heat demand of greenhouse $j$ at time step $k, H_{\mathrm{impEx} j}(k)$ denotes the imported heat by greenhouse $j$ from an external party at time step $k, \phi_{j}$ is the set of neighbors of greenhouse $j$, and $\alpha_{i j}$ denotes the percentage of heat loss due to transportation between greenhouse $i$ and $j$. Moreover, we have capacity constraints for the buffer and constraint for the amount of heat imported from external parties or exchanged between two neighbors due to for instance pipe or network capacity.

There is also an additional constraint for the transported heat among the greenhouses in order to make sure that the supply-demand balance is satisfied at each time step,

$$
\begin{aligned}
0 \leq B_{\mathrm{S} j}(k) & \leq B_{\mathrm{C} j} \forall k, j \\
0 \leq H_{\mathrm{impEx} j}(k) & \leq \overline{\mathrm{U}}_{\mathrm{impEx} j} \forall k, j \\
H_{\mathrm{exch} i j} & =-H_{\operatorname{exch} j i} \quad \forall j, i \in \phi_{j} \\
\underline{\mathrm{U}}_{\text {exch } i j} & \leq H_{\text {exch } i j} \leq \overline{\mathrm{U}}_{\text {exch } i j}
\end{aligned}
$$

where $\overline{\mathrm{U}}_{\mathrm{impEx} j}$ is the maximum possible heat import from external parties and $\underline{\mathrm{U}}_{\text {exch } i j}$ and $\overline{\mathrm{U}}_{\text {exchij }}$ are minimum and maximum amount of heat that can be exchanged between two adjacent neighbors. Note that $H_{\text {exch } i j}$ takes both positive and negative values indicating the imported heat by greenhouse $j$ from greenhouse $i$ and exporting heat from greenhouse $i$ to greenhouse $j$, respectively.

Figure 1 illustrates the energy flow between one greenhouse and the network of greenhouses, as well as the heatproducing external parties and the energy retailers.

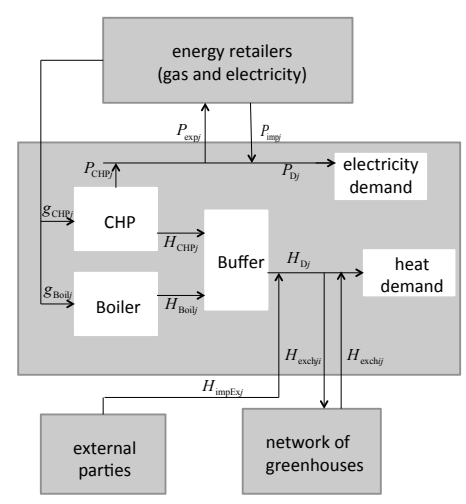

Fig. 1. Energy flow between greenhouse $j$, network of greenhouses, heat producing external parties, and energy retailers.

Remark 1: The connection between each greenhouse $j$ and the external parties in Figure 1 does not reflect the physical connection and is only an indicator for the heat flow.

\section{MODEL PREDICTIVE CONTROL FOR STGS}

Our aim is to reduce the overall production costs of the network while providing the network's required heat under different operational constraints such as the limits for the generators and the buffers. To this end, we intend to develop an advanced control approach that is suitable for practical applications. The control objective will be focused on demand response [9], [17], which is the ability of domestic net-consumption of heat to respond to real-time ${ }^{1}$ electricity prices. In this paper by "real-time" electricity prices we mean the hourly varying supply tariff, which is equal to the hourly day-ahead prices of the electricity market.

\footnotetext{
${ }^{1}$ The real-time electricity price is the one that varies almost every 15 minutes in the electricity market on the exact day of the electricity production.
} 
The control strategy that is proposed here for demand response is Model Predictive Control (MPC). The control objective is to minimize the total heat production costs, which includes the variable costs of the network related to the heat production as well as the earnings. Without loss of generality, we assume that the network is owned by a single owner and hence, all greenhouses cooperate with each other in order to keep the total heat generation costs of the network as low as possible. This means that they try to generate as much heat as possible in order to satisfy the heat demand of the network and buy as less as possible from the external parties. The total heat production cost function of greenhouse $j$ at time step $k$ can be defined as

$$
\begin{aligned}
C( & \left.P_{\mathrm{G}_{\mathrm{CHP}} j}(k), H_{\mathrm{G}_{\mathrm{Boil}} j}(k), H_{\mathrm{impEx} j}(k), \mu_{\mathrm{CHP} j}^{\text {start }}(k)\right) \\
= & C_{\mathrm{G}}\left(P_{\mathrm{G}_{\mathrm{CHP}} j}(k), H_{\mathrm{G}_{\mathrm{Boil}} j}(k)\right)+C_{\mathrm{O}}\left(P_{\mathrm{G}_{\mathrm{CHP}} j}(k)\right) \\
& +C_{\mathrm{imp}}\left(H_{\mathrm{impEx} j}(k)\right)+C_{\mathrm{start}}\left(\mu_{\mathrm{CHP} j}^{\text {start }}(k)\right)-E_{\mathrm{P}}\left(P_{\mathrm{CHP}, j}(k)\right) .
\end{aligned}
$$

The heat generation cost for each greenhouse depends on the amount of fuel that is used. Therefore, considering equations (1) and (2), it can be defined as

$$
C_{\mathrm{G}}\left(P_{\mathrm{G}_{\mathrm{CHP}} j}(k), H_{\mathrm{G}_{\mathrm{Boil}} j}(k)\right)=\left(g_{\mathrm{CHP} j}(k)+g_{\mathrm{Boil} j}(k)\right) F_{\text {price }} .
$$

For each CHP, there will also be an additional cost, namely, the operation cost, which is defined for each greenhouse $j$ at time step $k$ as

$$
C_{\mathrm{O}}\left(P_{\mathrm{G}_{\mathrm{CHP}} j}(k)\right)=P_{\mathrm{G}_{\mathrm{CHP}} j}(k) \cdot C_{\mathrm{CHP}} .
$$

The import cost matters when the greenhouse needs to buy heat from an external party, in the case that the generated heat by the greenhouse itself and the amount that is imported from other greenhouses in the network is less than its demand. The cost of importing heat by greenhouse $j$ at time step $k$ is

$$
C_{\mathrm{imp}}\left(H_{\mathrm{impEx} j}(k)\right)=H_{\mathrm{impEx} j}(k) \cdot H_{\text {buyingEx }}(k),
$$

where $H_{\text {buyingEx }}(k)$ is the price that greenhouse $j$ pays for buying heat from external parties at time step $k$. We assume that the taxes and the transportation cost are included in $H_{\text {buyingEx }}(k)$. Moreover, there are fixed start-up costs and fuel-based start-up costs for a CHP unit of greenhouse $j$, which can be calculated as [8]

$$
C_{\text {start }}\left(\mu_{\mathrm{CHP} j}^{\text {start }}(k)\right)=\mu_{\mathrm{CHP} j}^{\text {start }}(k)\left(C_{\text {fix }}+g_{\text {start }} \cdot F_{\text {price }}\right),
$$

where $\mu_{u j}^{\text {start }}$ is a binary variable such that $\mu_{u j}^{\text {start }}(k)=1$ if unit $u$ (CHP or boiler) of greenhouse $j$ is started for production of energy at time step $k$ and $\mu_{u j}^{\text {start }}(k)=0$ otherwise. The second part of the production cost is related to the electricity earnings obtained from selling electricity to the electricity market. The selling price is variable and is different every hour. The electricity earnings of greenhouse $j$ at time step $k$ can be written as

$E_{\mathrm{P}}\left(P_{\mathrm{G}_{\mathrm{CHP}} j}(k)\right)= \begin{cases}\left(P_{\mathrm{G}_{\mathrm{CHP}} j}(k)-P_{\mathrm{D} j}(k)\right) P_{\text {selling }}(k) \\ & \text { if } P_{\mathrm{G}_{\mathrm{CHP}} j}(k) \geq P_{\mathrm{D} j}(k) \\ 0 & \text { if } P_{\mathrm{G}_{\mathrm{CHP}} j}(k)<P_{\mathrm{D} j}(k)\end{cases}$

where $P_{\mathrm{D} j}(k)$ indicates the electricity demand of greenhouse $j$ at time step $k$ and $P_{\text {selling }}(k)$ is the selling price of electricity at time step $k$. Note that since we assume cooperation between the greenhouses, there are no heat earnings while the greenhouses exchange heat among each other.

Therefore, considering (10), the cost function $J(k)$ at time step $k$ over the prediction horizon $N_{\mathrm{p}}$ is defined as

$$
\begin{aligned}
J=\sum_{l=0}^{N_{\mathrm{p}}-1} \sum_{j=1}^{n} C\left(P_{\mathrm{G}_{\mathrm{CHP}} j}(k+l), H_{\mathrm{G}_{\mathrm{Boil}} j}(k+l),\right. \\
\left.H_{\mathrm{impEx} j}(k+l), \mu_{\mathrm{CHP} j}^{\text {start }}(k+l)\right) .
\end{aligned}
$$

This cost function will be minimized subject to the constraints on different components of the systems. Some of these constraints have been presented in the previous section. In addition to those, we need extra constraints related to onoff states of the CHP and boiler [9]. We define $\mu_{\mathrm{u}, j}^{\text {stop }}$ as a binary variable such that $\mu_{\mathrm{u}, j}^{\text {stop }}(k)=1$ if unit $u$ (CHP or boiler) of greenhouse $j$ is shut down at time step $k$ and 0 otherwise. Moreover, we define the binary variable $v_{\mathrm{u} j}(k)$ for each production unit $u$, of greenhouse $j$ at time step $k$ such that $v_{\mathrm{u} j}(k)=1$ if unit $u$ operates and 0 otherwise. Therefore, the capacity constraints for the heat production, i.e., equations (3)-(4) can be rewritten as

$$
\underline{\mathrm{U}}_{\mathrm{u} j} \cdot v_{\mathrm{u} j}(k) \leq H_{G_{\mathrm{u}} j}(k) \leq \overline{\mathrm{U}}_{\mathrm{u} j} \cdot v_{\mathrm{u} j}(k) \forall k, j
$$

Moreover, the following equations link the above binary variables [8], [9]:

$$
\begin{aligned}
& v_{\mathrm{u} j}(k)-v_{\mathrm{u} j}(k-1)=\mu_{\mathrm{u}, j}^{\text {start }}(k)-\mu_{\mathrm{u}, j}^{\text {stop }}(k) \forall j, k \\
& \mu_{\mathrm{u}, j}^{\text {start }}(k)+\mu_{\mathrm{u}, j}^{\text {stop }}(k) \leq 1 \forall j, k \\
& v_{\mathrm{CHP} j}(k) \geq \sum_{k-\underline{\mathrm{U}}_{\mathrm{CHP} j}^{o n}<\ell \leq k} \mu_{\mathrm{CHP}, j}^{\text {start }}(\ell) \forall j, k \\
& 1-v_{\mathrm{CHP} j}(k) \geq \sum_{k-\underline{\mathrm{U}}_{\mathrm{CHP} j}^{o f f}<\ell \leq k} \mu_{\mathrm{CHP}, j}^{\text {stop }}(\ell) \forall j, k
\end{aligned}
$$

The last two constraints are related to the minimum time-on and time-off constraints for each CHP unit ${ }^{2}$ where $\underline{\mathrm{U}}_{\mathrm{CHP} j} \mathrm{j}$ and $\underline{\mathrm{U}}_{\mathrm{CHP} j}^{o f f}$ are minimum required on and off time steps respectively.

In order to obtain a linear system with continuous and binary variables, we apply the mixed logical dynamical (MLD) formalism [3], which allows the transformation of logical statements involving continuous variables into mixed-integer linear inequalities. Accordingly, we can rewrite (15) as a linear equation by introducing new binary and continuous

\footnotetext{
${ }^{2}$ As mentioned by experts at Eneco, a Dutch utility company and our project sponsor, boilers do not require a time-on/off constraints.
} 
auxiliary variables. In this way, the system dynamics and the constraints are formulated as mixed-integer linear equations and hence, we solve a mixed-integer linear programming (MILP) problem. Note that this control approach is a centralized one, which means while the overall production cost of the network is minimized, each individual greenhouse may not have the optimal cost at each time step.

\section{SOLVING THE WORST-CASE MPC}

At the beginning of each time step $k$, the controller measures the system state of the previous step. In our case, the state variables are $B_{\mathrm{S} j}, v_{\mathrm{CHP} j}$, and $v_{\mathrm{Boil} j}$. At each time step $k$, we assume that the previous value of these variables is known or measured. Then, using the information regarding the demand and the energy price, the controller determines the decision variables $P_{\mathrm{G}_{\mathrm{CHP}} j}, H_{\mathrm{G}_{\mathrm{Boil}} j}, H_{\mathrm{impEx} j}, \mu_{\mathrm{u} j}^{\text {stop }}$, and $\mu_{\mathrm{u} j}^{\text {start }}$. We choose 24 time steps, i.e., $k=1, \ldots, 24$, corresponding to the 24 hours in one day.

We also assume that there is an uncertainty in the heat demand $H_{\mathrm{D}}$, i.e., $H_{\mathrm{D}}(k)=H_{\mathrm{D} \text {,pred }}(k)+e(k)$ where $H_{\mathrm{D} \text {,pred }}(k)$ is the predicted heat demand for the greenhouses at time step $k$. We gather the uncertainty for time steps $k, \ldots, k+N_{\mathrm{p}}-1$ in the vector $\tilde{e}(k)=\left[e^{T}(k), \ldots, e^{T}\left(k+N_{\mathrm{p}}-1\right)\right]^{T} \in \mathscr{E}$ where $\mathscr{E}=$ $\{\tilde{e}(k): \tilde{S} \tilde{e}(k) \leq \tilde{q}\}$ is a bounded polyhedral set. Accordingly, we can define the worst-case MPC optimization problem as

$$
\begin{aligned}
& \min _{\tilde{u}(k)} \max _{\tilde{e}(k) \in \mathscr{E}} J(\tilde{u}(k), \tilde{e}(k)) \\
& \text { s.t. } P(k) \tilde{u}(k)+Q(k) \tilde{e}(k)+q(k) \leq 0
\end{aligned}
$$

where $J$ is the cost function, $\tilde{u}(k)$ is the vector of decision variables containing both continuous and binary variables as well as the continuous and binary auxiliary variables obtained from the MLD model (defined similarly to $\tilde{e}(k)$ ), $P(k), Q(k)$ are inequality constraint matrices and $q(k)$ is the inequality constraint constant vector, all defined according to the constraints (6)-(9) and (17)-(21). Since both the cost function $J$ and the constraints are piecewise affine in $\tilde{u}(k)$, we can solve the optimization problem (22)-(23) as an MILP problem.

Remark 2: Solving an MILP for the robust MPC design does not scale well with the complexity (size) of the model/system, and disturbance set representation. This issue can be avoided by either decreasing the number of binary variables by choosing a small-enough prediction horizon $N_{\mathrm{p}}$, or by relaxing the constraints on the binary variables to obtain an LP optimization problem instead. Generally, MILP complexity grows exponentially as the number of binary variables increases.

The first approach we apply to solve our MILP optimization problem is multi-parametric MILP (mp-MILP) optimization. To this end, we solve the inner optimization problem first. For a given $\tilde{u}(k)$, the optimization problem

$$
\begin{aligned}
& \max _{\tilde{e}(k)} J(\tilde{u}(k), \tilde{e}(k)) \\
& \text { s.t. } \tilde{S} \tilde{e}(k) \leq \tilde{q} \\
& \quad P(k) \tilde{u}(k)+Q(k) \tilde{e}(k)+q(k) \leq 0
\end{aligned}
$$

can be solved as an mp-MILP problem, in which $\tilde{u}(k)$ is the parameter, using the algorithm in [6].

Let $\tilde{e}^{*}(\tilde{u}(k))=\arg \max _{\tilde{e}(k)} J(\tilde{u}(k), \tilde{e}(k))$ denote the solution of the mp-MILP problem (24)-(25), which is a piecewiseaffine function in $\tilde{u}(k)$ (see [4], [11]). Hence, the outer optimization problem, i.e.,

$$
\begin{aligned}
& \min _{\tilde{u}(k)} J\left(\tilde{u}(k), \tilde{e}^{*}(\tilde{u}(k))\right) \\
& \text { s.t. } P(k) \tilde{u}(k)+Q(k) \tilde{e}^{*}(\tilde{u}(k))+q(k) \leq 0
\end{aligned}
$$

can be solved as an MILP optimization problem using the available MILP solvers that are based on e.g. branch-andbound or cutting plane algorithms [2].

Note that the available mp-MILP algorithms are not very efficient when the size of the vector of parameters and the prediction horizon $N_{\mathrm{p}}$ increases. Therefore, we now discuss alternative approaches to $\mathrm{mp}$ MILP. One approach is to use Monte Carlo simulation to eliminate the inner optimization problem as follows. Let $\tilde{e}^{(1)}(k), \ldots, \tilde{e}^{(M)(k)}$ denote $M$ different noise realizations belonging to the polyhedral set $\mathscr{E}$ and let $t(k)=$ $\max _{\tilde{e}^{(1)}(k), \ldots, \tilde{e}^{(M)(k)}}\left(J\left(\tilde{u}(k), \tilde{e}^{(1)}(k)\right), \cdots, J\left(\tilde{u}(k), \tilde{e}^{(M)}(k)\right)\right)$. The optimization problem (22)-(23) can be then rewritten as

$$
\begin{array}{ll}
\min _{\tilde{u}(k), t(k)} t(k) \\
\text { s.t. } t(k) \geq J\left(\tilde{u}(k), \tilde{e}^{(1)}(k)\right) \\
\quad \cdots \\
t(k) \geq J\left(\tilde{u}(k), \tilde{e}^{(M)}(k)\right) \\
& P(k) \tilde{u}(k)+Q(k) \tilde{e}^{(1)}(k)+q(k) \leq 0 \\
& \cdots \\
& P(k) \tilde{u}(k)+Q(k) \tilde{e}^{(M)}(k)+q(k) \leq 0
\end{array}
$$

which can be solved as an MILP optimization problem.

Another approach is to use the Farkas' lemma [5] to obtain an MILP optimization problem that is equivalent to (22)-(23). Let $J(\tilde{u}, \tilde{e})=C_{1}^{T} \tilde{u}(k)+C_{2}^{T} \tilde{e}(k)$ where $C_{1}$ and $C_{2}$ are vectors of coefficients. For a fixed value of $t$, we can rewrite (22)-(23) as

$$
\begin{aligned}
& \min _{\tilde{u}(k)} C_{1}^{T} \tilde{u}(k)+t \\
\text { s.t. } & C_{2}^{T} \tilde{e}(k) \leq t \quad \forall \tilde{e}(k): S \tilde{e}(k) \leq \tilde{q} \\
& P(k) \tilde{u}(k)+Q(k) \tilde{e}(k)+q(k) \leq 0
\end{aligned}
$$

Now, using Farkas' lemma, we can rewrite the constraints as

$$
\begin{gathered}
C_{2}^{T} \tilde{e}(k)-t=\mu^{T}(S \tilde{e}(k)-\tilde{q})+\alpha \\
P_{i}(k) \tilde{u}(k)+Q_{i}(k) \tilde{e}(k)+q_{i}(k)=\beta_{i}+\Lambda_{i}(S \tilde{e}(k)-\tilde{q}) \\
i=1, \ldots, m \\
\Lambda, \mu \geq 0, \quad \alpha, \beta \leq 0
\end{gathered}
$$

where $\mu$ and $\Lambda$ are Lagrange multipliers such that $\mu$ is a vector of size $\tilde{q}, \Lambda$ is a matrix of size $m \times n_{\tilde{q}}$ and $n_{\tilde{q}}$ is the size of vector $\tilde{q}$. Let $m$ denote the number of constraints in (23), $\beta$ is a vector of length $m$, and $\alpha$ is a scalar. So we can rewrite (30)-(32) as

$$
\min _{\tilde{u}, \mu, \Lambda, \alpha, \beta} C_{1}^{T} \tilde{u}+t
$$




$$
\begin{array}{ll}
\text { s.t. } & C_{2}=S^{T} \mu \\
& t=\mu^{T} \tilde{q}-\alpha \\
& Q_{i}(k)=S^{T} \Lambda_{i} \quad i=1, \ldots, m \\
& P_{i}(k) \tilde{u}(k)+q_{i}(k)=\beta_{i}-\Lambda_{i} \tilde{q} \quad i=1, \ldots, m \\
& \Lambda, \mu \geq 0, \quad \alpha, \beta \leq 0
\end{array}
$$

which is an MILP optimization problem. This problem will be solved for different values of $t$ and then, we choose the optimal $\tilde{u}$ that have the minimum objective function $C_{1}^{T} \tilde{u}+t$ for all these values of $t$. Note that since in our model for the STG, the cost function is not explicitly dependent on $\tilde{e}(k)$, we can solve the optimization problem (36)-(41) by eliminating $t$ and the constraints (37) and (38).

\section{EXAMPLE}

In this section, we solve robust MPC optimization problem to obtain a day-ahead prediction for the heat production plan for a small network of greenhouses and we compare the results with the ones obtained using robust optimal control approach. In this case study, we consider two greenhouses and an external producer. Each of the greenhouses has a CHP unit, a boiler, and a buffer. The aim is to minimize the heat production cost of the network while satisfying the network constraints and the supply-demand balance.

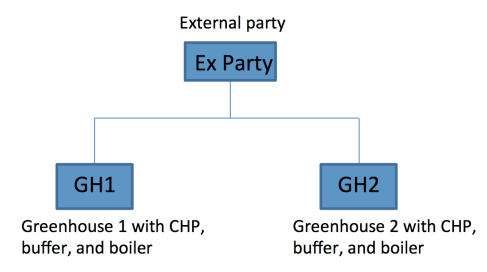

Fig. 2. Physical topology of the thermal network of the case study

We consider the cost function (16) and we an uncertain heat demand $H_{\mathrm{D} j}(k)=H_{\mathrm{D}, \text { pred } j}(k)+e_{j}(k)$ where $H_{\mathrm{D}, \operatorname{pred} j}(k)$ is the predicted heat demand for greenhouse $j \in\{1,2\}$ at time step $k$ and $e_{j}(k)$ denotes the uncertainty such that $\left|e_{j}(k)\right| \leq 1$. We also have $\underline{\mathrm{U}}_{\mathrm{CHP} j}^{o n}=5$ and $\underline{\mathrm{U}}_{\mathrm{CHP} j}^{o f f}=3$.

The cost function is minimized subject to the constraints (6)-(9) and (17)-(21). Using MPC with a prediction horizon $N_{\mathrm{p}}=15$, at each time step we have 285 control variables (150 binary variables including the auxiliary variables from the MLD model), an uncertainty vector of size 30, and 660 inequality constraints. In the optimal control approach since we optimize the system for 24 hours, we have 456 control variables (240 binary variables), an uncertainty vector of size 48, and 1056 inequality constraints. For both approaches, we apply the three different methods explained in Section IV. Even though we picked a very small network to be able to use mp-MILP approach, solving the mp-MILP optimization problem using the MPT toolbox seems to be very inefficient for a problem of this size. Even for a small prediction horizon such as $N_{\mathrm{p}}=3$, the computation time is so long that makes this approach infeasible for this example. Therefore, we only use the Monte Carlo approach and the equivalent reformulation of min-max problem based on Farkas' lemma (cf. Section IV) to obtain the day-ahead heat production plan, which is solved in Matlab R2014b on a $2.6 \mathrm{GHz}$ Intel Core i5 processor. To solve the optimization problem (28)-(29), we chose $M=500$ different uncertainty vectors $e$ to obtain a $0.95 \%$ confidence level with accuracy error of $1 \%$ and we use the MILP solver from IBM CPLEX. The computation time and the total heat production cost of the network during 24 hours are given in Table II. The total production costs

\begin{tabular}{|c|c|c|c|}
\hline $\begin{array}{c}\text { Control } \\
\text { Approach }\end{array}$ & $\begin{array}{c}\text { Solution } \\
\text { Approach }\end{array}$ & $\begin{array}{c}\text { Time } \\
(\mathrm{s})\end{array}$ & $\begin{array}{c}\text { Production Cost } \\
(€)\end{array}$ \\
\hline Robust MPC & Monte Carlo & 3180 & 22576 \\
\hline & Farkas' lemma & 116 & 22325 \\
\hline Robust Optimal Control & Monte Carlo & 207 & 23190 \\
\hline & Farkas' lemma & 13 & 22708 \\
\hline
\end{tabular}

TABLE II

COMPUTATION TIME AND TOTAL PRODUCTION COST FOR THE NETWORK

of this network for the case that the greenhouses do not exchange heat among each other and only buy from the external heat producers is $30194 €$. The choice between the MPC approach and the optimal control approach is a matter of trade-off between the computation time and the production cots. The MPC computation time can be reduced by choosing a smaller prediction horizon $N_{\mathrm{p}}$. In this example we have chosen $N_{\mathrm{p}}=15$ to show that even for a large prediction horizon, this approach is still computationally feasible.

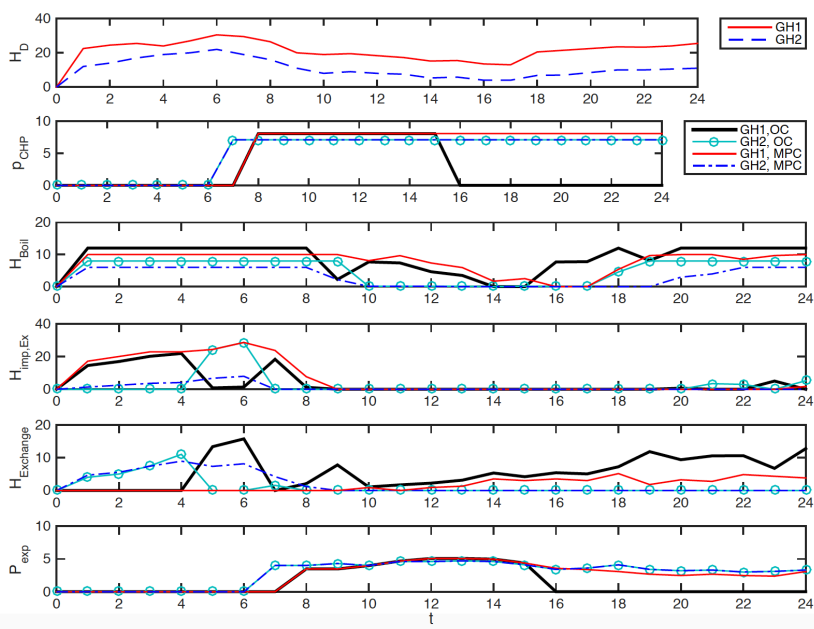

Fig. 3. Heat and electricity production plan of the thermal network using robust MPC approach vs. robust optimal control (OC) approach.

The optimization results using robust optimal control versus the robust MPC, solved using the reformulation technique based on Farkas' lemma, are shown in Figure 3. The first plot of Figure 3 shows the heat demand of each greenhouse for one day. The second and third plots show the amount of electricity and heat that needs to be generated by the $\mathrm{CHP}$ and boiler units at each greenhouse, respectively. The forth plot shows the amount of imported heat from the 
external parties. The fifth plot illustrates the amount of heat that is imported by each greenhouse from the other one. The last plot shows the amount of electricity that each greenhouse can export to the electricity market. Here, we assume that the production capacity of the CHP and boiler units are similar to each other for the sake of illustration; in reality, the boilers capacities are much higher and since they impose less production costs, they will be used more than the CHPs. Hence, in practice, CHPs are mainly used when the electricity price is high so the greenhouses can benefit from selling the extra generated electricity to the electricity market. Here also, the CHPs are mainly used during the hours that the electricity price is quite high and they can also sell the extra electricity in the market (cf. second, third, and last plot is Figure 3). Moreover, since the greenhouses can exchange heat among each other, they only need to import heat from the external party in the early hours of the day and for the rest, they receive heat from each other (cf. forth and fifth plot in Figure 3).

Based on this case study, we can conclude that the current network setting is more cost-efficient compared to the setting with a single (main) producer where the greenhouses act as a costumer rather than being a prosumer. Moreover, robust MPC is a better control choice than robust optimal control although it requires more computation time. In the former approach, the control input will be updated and hence, the controller performs better in the presence of the new information, while in the latter, the optimal control input is obtained for the entire simulation period ( 24 hours) and hence, it is only based on the initial information from the network.

\section{CONCLUSIONS}

We have considered control of a typical smart thermal grid, namely a network of greenhouses, under uncertainties in demand and/or response. Our aim was to provide a dayahead heat production plan for the network assuming there is uncertainty in the heat demand. To obtain an economical plan, we minimized the total heat production cost of the network using model predictive control. We assumed the uncertainty to be bounded and hence, a worst-case MPC optimization problem was solved. Since both the cost function and the constraints are linear, the optimization problem was formulated as a mixed-integer linear programming (MILP) problem; we have discussed three approaches to solve the obtained optimization problem. In a case study, we compared the MPC approach with the optimal control approach to obtain a day-ahead production plan for a sample network of greenhouses. Based on this example, robust MPC performs better than robust optimal control although it requires more computation time.

An alternative scenario to the centralized control architecture is that each greenhouse tries to maximize its own benefit and hence, they will sell heat to the other greenhouses in the network. The efficient control approach in this case is a distributed model predictive control approach in which the agents can only have partial information about the network. Moreover, in future work, we will also consider the constraints of the physical network's model to be able to take the dependencies and the possible delays into account.

\section{ACKNOWLEDGMENTS}

Research partially funded by the Dutch utility company Eneco, and by the Netherlands Organization for Scientific Research (NWO) under the project Aquifer Thermal Energy Storage Smart Grids (ATES-SG), grant number 408-13-030. Moreover, the authors would like to thank Dr. Mathijs de Weerdt and Dr. Matthijs Spaan from Delft University of Technology and the collaborators from AgroEnergy.

\section{REFERENCES}

[1] A. Arteconi, N. J. Hewitt, and F. Polonara. Domestic demand-side management (DSM): Role of heat pumps and thermal energy storage (TES) systems. Applied Thermal Engineering, 51(1-2):155-165, 2013.

[2] A. Atamtürk and M. W. P. Savelsbergh. Integer-programming software systems. Annals of Operations Research, 140(1):67-124, November 2005.

[3] A. Bemporad, F. Borrelli, and M. Morari. Model predictive control based on linear programming - The explicit solution. IEEE Transactions on Automatic Control, 47(12):1974-1985, December 2002.

[4] F. Borrelli. Constrained Optimal Control of Linear and Hybrid Systems, volume 290 of Lecture Notes in Control and Information Sciences. Springer, Berlin, Germany, 2003.

[5] S. Boyd and L. Vandenberghe. Convex Optimization. Cambridge University Press, Cambridge, UK, 2004.

[6] V. Dua and E. N. Pistikopoulos. An algorithm for the solution of multiparametric mixed integer linear programming problems. Annals of Operations Research, 99(1-4):123-139, 2000.

[7] S. S. Farahani, T. van den Boom, and B. De Schutter. Model predictive control for stochastic max-min-plus-scaling systems - An approximation approach. In Proceedings of the 50th IEEE Conference on Decision and Control and European Control Conference (CDCECC), pages 391-396, Orlando, USA, December 2011.

[8] E. Friis-Jensen. Modeling of the combined heat and power system of greater copenhagen. Master's thesis, Technical University of Denmark, 2010.

[9] M. Houwing, R.R. Negenborn, and B. De Schutter. Demand response with micro-CHP systems. Proceedings of the IEEE, 99(1):200-213, 2011.

[10] P. J. Immonen. Mathematical models in cogeneration optimization. Instrument Society of America POWID Newsletter, December 2000.

[11] E. C. Kerrigan and D. Q. Mayne. Optimal control of constrained, piecewise affine systems with bounded disturbances. In Proceedings of the 41st IEEE Conference on Decision and Control, pages 15521557, Las Vegas, Nevada, December 2002.

[12] P. Mc Namara, R. R. Negenborn, B. De Schutter, and G. Lightbody. Optimal coordination of a multiple HVDC link system using centralized and distributed control. IEEE Transactions on Control Systems Technology, 21(2):302-314, March 2013.

[13] R. R. Negenborn, A. G. Beccuti, T. Demiray, S. Leirens, G. Damm, B. De Schutter, and M. Morari. Supervisory hybrid model predictive control for voltage stability of power networks. In Proceedings of the 2007 American Control Conference, pages 5444-5449, New York, NY, July 2007.

[14] S. Ramchurn, P. Vytelingum, A. Rogers, and N. R. Jennings. Agentbased control for decentralized demand side management in the smart grid. In Proceedings of the 10th International Conference on Autonomous Agents and Multiagent Systems, Taipei, Taiwan, May 2011.

[15] J. B. Rawlings and D. Q. Mayne. Model Predictive Control: Theory and Design. Nob Hill Publishing, Madison, Wisconsin, 2009.

[16] M. Ristic, D. Brujic, and K. Thoma. Economic dispatch of distributed combined heat and power systems participating in electricity spot markets. Proceedings of the Institution of Mechanical Engineers, Part A: Journal of Power and Energy, 222(7):743-752, January 2008.

[17] M. Roozbehani, M. A. Dahleh, and S. K. Mitter. Volatility of power grids under real-time pricing. IEEE Transactions on Power Systems, 27(4):1926-1940, 2012. 